\title{
DÜBLIN
}

Technological University Dublin

ARROW@TU Dublin

\section{Block-based Classification Method for Computer Screen Image Compression}

\author{
Kai Wu \\ Technological University Dublin \\ Richard Gahan \\ Technological University Dublin \\ Patrick O'Friel \\ Technological University Dublin, patrick.ofriel@tudublin.ie
}

Follow this and additional works at: https://arrow.tudublin.ie/engscheleart

Part of the Electrical and Computer Engineering Commons

\section{Recommended Citation}

K. Wu, R. Gahan and P. O'Friel, "Block-based Classification Method for Computer Screen Image Compression," 2018 29th Irish Signals and Systems Conference (ISSC), 2018, pp. 1-6, doi: 10.1109/ ISSC.2018.8585352.

This Conference Paper is brought to you for free and open access by the School of Electrical and Electronic Engineering at ARROW@TU Dublin. It has been accepted for inclusion in Conference papers by an authorized administrator of ARROW@TU Dublin. For more information, please contact arrow.admin@tudublin.ie, aisling.coyne@tudublin.ie,gerard.connolly@tudublin.ie.

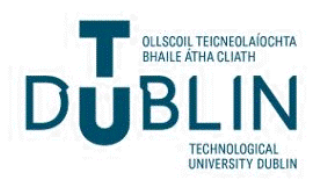




\section{Block-based Classification Method for Computer Screen Image Compression}

\author{
Kai Wu \\ Department Electronic Engineer \\ Institute of Technology Tallaght \\ Tallaght, Dublin, Ireland \\ Email: kevinhu0010@gmail.com
}

\author{
Richard Gahan \\ Department Electronic Engineer \\ Institute of Technology Tallaght \\ Tallaght, Dublin, Ireland \\ Email: richard.gahan@ittdublin.ie
}

\author{
Patrick O'Friel \\ Department Electronic Engineer \\ Institute of Technology Tallaght \\ Tallaght, Dublin, Ireland \\ Email: Patrick.OFriel@ittdublin.ie
}

\begin{abstract}
In this paper, a high accuracy and reduced processing time block based classification method for computer screen images is presented. This method classifies blocks into five types: smooth, sparse, fuzzy, text and picture blocks. In a computer screen compression application, the choice of block compression algorithm is made based on these block types. The classification method presented has four novel features. The first novel feature is a combination of Discrete Wavelet Transform (DWT) and colour counting classification methods. Both of these methods have only been used for computer image compression in isolation in previous publications but this paper shows that combined together more accurate results are obtained overall. The second novel feature is the classification of the image blocks into five block types. The addition of the fuzzy and sparse block types make the use of optimum compression methods possible for these blocks. The third novel feature is block type prediction. The prediction algorithm is applied to a current block when the blocks on the top and the left of the current block are text blocks or smooth blocks. This new algorithm is designed to exploit the correlation of adjacent blocks and reduces the overall classification processing time by $33 \%$. The fourth novel feature is down sampling of the pixels in each block which reduces the classification processing time by $62 \%$. When both block prediction and down sampling are enabled, the classification time is reduced by $74 \%$ overall. The overall classification accuracy is $98.46 \%$.
\end{abstract}

Index Terms-compound image, block-based classification, block type prediction, classification time, accuracy

\section{INTRODUCTION}

In a thin client application, the computer screen image at the server is compressed and sent to the thin client. The server computer screen image is usually made up from various types of displayed content, for example Web pages, PDF files, slides, text, graphics and pictures [9], see Fig.1. This is refered to as a compound image. The different regions of the compound image have different requirements in how they can be compressed. Text regions require high quality or lossless compression whereas picture regions can use a lossy compression algorithm without noticeable visual impact on the user. To determine which compression algorithm to use, the different regions of the compound image must be classified. In general, there are two methods to determine the different region types: layers based [1] and block based

978-1-5386-6046-1/18/\$31.00 (C)2018 IEEE

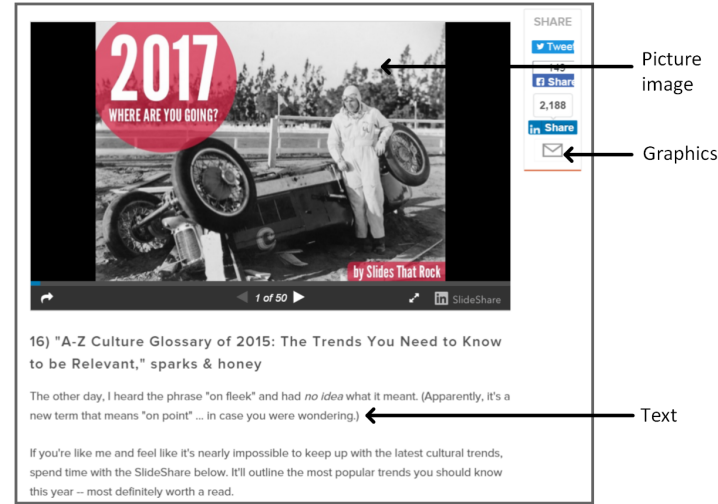

Figure 1. Example of a compound image

[2] classification. This paper presents a block based approach to classification of the compound image, see Fig.2. In this method, the image is segmented into blocks of pixels and each block is then classified into one of the five types: smooth, sparse, fuzzy, picture and text. An optimum compression algorithm can then be selected for each of the five types. In this paper, novel methods are used to improve the classification accuracy compared to published results [8] and to reduce the overall classification processing time.

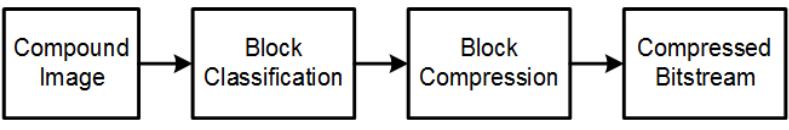

Figure 2. Block-based compound compression system

\section{RELATED WORKS}

In [6], Lin classified each $16 \times 16$ non-overlapping block into text/graphic blocks and picture blocks by counting the number of colours in each block. A colour number threshold value $T=32$ is set. This is a coarse classification, a refinement process is then applied after this step (if $T>32$ ) to extract text and graphic pixels from picture blocks. If refinement is applied to all blocks, it is time-consuming. Therefore refinement is only applied to picture blocks which are classified by coarse classification. If there are large picture regions in a 
compound image, the refinement process is applied to most blocks and it is time consuming. The method proposed in [6] can classify many smooth blocks as text/graphic blocks which are compressed by a lossless encoder, i.e., LZW. The addition of the smooth block type used in this paper allows for the selection of a more suitable compression algorithm, e.g., sending the colour value directly.

In [7], Wenpeng Ding proposed a classification algorithm based on two features: histogram and gradient of the current block. It calculates the gradient of each pixel, counts the number of colours and does a classification for each block. All blocks are classified into four types: smooth, text, hybrid and picture blocks. This method is a variation of the colour counting technique.

In [15], Zhaotai Pan proposed a feature-based block-type classification method. He selected two features for classification: the number of high gradient pixels and the number of basic colours. Different threshold values are set according to the type of block around the current block. The classification method in [15] is context-adaptive. The method proposed in this paper is also context-adaptive.

In [8], Ebenezer and Jemi proposed a method for compound image classification based on DWT. They applied singlelevel wavelet decomposition to each block and generate four different sub-bands, each of them representing one feature of the current block. Three of the four sub-bands are used to classify the type of blocks. However the classification method in [8] does not work well in compound images with large regions of pictures. Some picture blocks that contain tree branches or complex buildings or wire fences can be classified as text blocks because these objects also have horizontal, slanted and vertical lines. The method proposed in this paper uses DWT in conjunction with colour counting to overcome this limitation.

In [14], Shen utilizes a histogram of each block and the number of isolated pixels to do classification. Three block types are used: picture block, text block and hybrid block. Smooth regions are treated as text blocks.

In [16], Qiu proposed an approach for classifying the screen image into two types of blocks: text and not-text block. They found that text often exists in a screen as a text region that spreads through many continuous blocks. Having two block types in [16] limits the selection of compression algorithm compared to five block types presented in this paper.

\section{METHODOLOGY}

\section{A. Classificaiton with DWT Method}

The DWT method applies filters to an image to decompose it into four different sub-bands: LL, LH, HL and HH [8]. LL is an approximation of the original image, low-pass filtered in horizontal and vertical directions and subsampled by a factor of two. LH is low-pass filtered in horizontal direction and high-pass filtered in vertical direction. HL is high-pass filtered in horizontal direction and low-pass filtered in vertical direction. $\mathrm{HH}$ is high-pass filtered in both horizontal and vertical directions [10].
This feature of the DWT can be used to classify different type blocks. Text blocks usually have a higher frequency in the horizontal, vertical and diagonal directions compared to picture blocks because of colour transition between text and background. The standard deviation measure reflects the fluctuation of a group of data and the standard deviation values of text block sub-bands are usually greater than picture subbands. Thus, the standard deviation of sub-bands LH, HL and $\mathrm{HH}$ is calculated to indicate the type an image block.

The input image is first divided into non-overlapping blocks (size of $16 \times 16$ ). Each block is decomposed into LH, HL, HH sub-bands, and the standard deviation of each sub-band is computed according to equation (1). $\mu$ and $\sigma$ are two statistical features: mean and standard deviation.

$$
\begin{aligned}
\mu_{\theta} & =\sqrt{\frac{1}{W * H} \sum_{i=1}^{W} \sum_{j=1}^{H}\left[I_{\theta}(i, j)\right]^{2}} \\
\sigma_{\theta} & =\sqrt{\frac{1}{W * H} \sum_{i=1}^{W} \sum_{j=1}^{H}\left[I_{\theta}(i, j)-\mu_{\theta}\right]^{2}}
\end{aligned}
$$

Here $\theta \in\{L H, H L, H H\}, \mathrm{W}$ and $\mathrm{H}$ are the width and height in pixels of sub band $\theta, I_{\theta}(i, j)$ is the wavelet coefficient in sub band $\theta$. The Standard deviation of sub-bands LH, HL and $\mathrm{HH}, \sigma_{L H}, \sigma_{H L}$ and $\sigma_{H H}$, is used to classify the current block into different types according to the algorithm shown in Fig.3. Threshold values $T 1, T 2$ and $T 3$ are context adaptive, their values depend on the block types surrounding the current block.

\section{B. Block Types and Block Size}

Based on experiments, block size is set to be $16 \times 16$. The block size $16 \times 16$ is small enough to avoid hybrid blocks (e.g., a block contains text and picture pixels simultaneously) which are hard to compress. The block size $16 \times 16$ is also sufficiently large such that if a $16 \times 16$ block is down sampled to $8 \times 8$, the down-sampled block will retain the basic texture characteristic of original block.

Blocks are classified into five types: smooth, sparse, fuzzy, text and picture blocks. Appropriate compression algorithms can subsequently be applied to these five different block types to achieve a high compression ratio with little visual quality impact.

1) Smooth Block: There is only one colour in smooth block and this type of block is easy to compress, e.g., sending the colour value to the receiver directly.

2) Sparse Block: Sparse block, see Fig.4, means there are only a few elements in this block, e.g., some simple graphics shapes. These blocks can be compressed efficiently with some lossless (e.g., Run Length Encoding) compression algorithms. Sparse block classification is novel to this paper.

Based on experiments with sparse blocks, it is found that there is one dominant standard deviation. This means one of $\sigma_{L H}, \sigma_{H L}$ and $\sigma_{H H}$ is much bigger than the other two values. For example, $\sigma_{L H}>4.5 \times \sigma_{H L}$ and $\sigma_{L H}>4.5 \times \sigma_{H H}$. The 


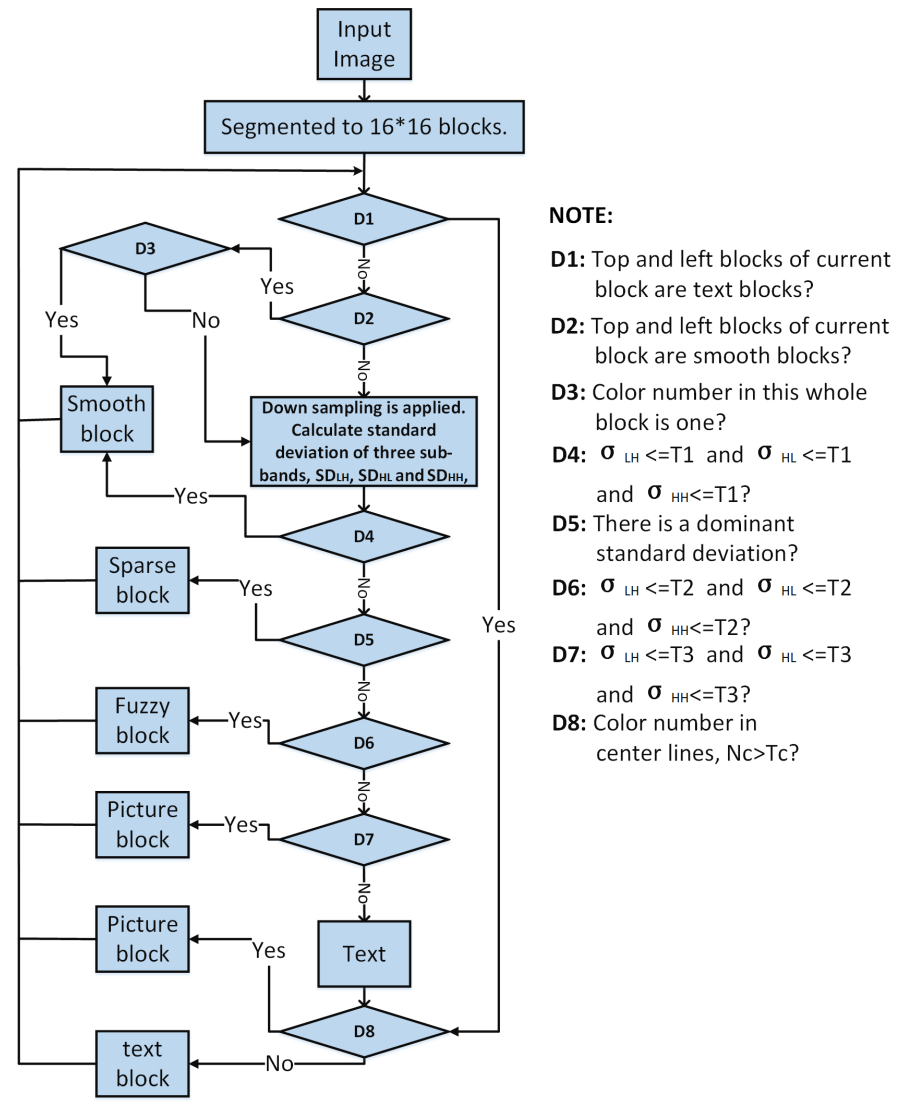

Figure 3. Framework of proposed classification system
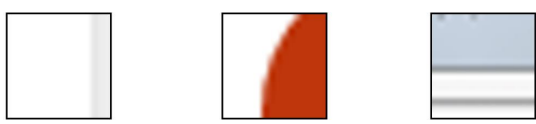

Figure 4. Examples of some amplified sparse blocks

factor of 4.5 is found from experiment to lead to accurate classification of sparse blocks.

3) Fuzzy Block: A Fuzzy block, see Fig.5, is a block which looks fuzzy to the human eyes. These blocks often exist in a natural landscape image, e.g., a waterfall, tree leaves, concrete ground, animal's hair or snow. These type of blocks can be compressed by low quality lossy algorithms (e.g., low quality JPEG) or algorithms suitable for continuous tone images (e.g., IW44 algorithm). Fuzzy block classification is another novel feature presented in this paper.
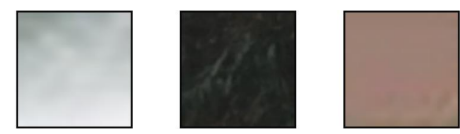

Figure 5. Some examples of amplified fuzzy blocks.

4) Text Block: Text has sharp edges and the human visual system is sensitive to quality loss of text elements. Therefore, text blocks need lossless image compression. If any apprecia-
Table I

TEXT BLOCKS CORRELATION TEST RESULT.

\begin{tabular}{|c|c|c|}
\hline$N_{\text {text }}$ & $N_{\text {prediction }}$ & Ratio (\%) \\
\hline 12530 & 6208 & 49.55 \\
\hline
\end{tabular}

ble text is detected in a block that block will be classified as a text block to preserve the text image quality.

5) Picture Block: A Picture block only contains picture elements. Usually a picture block can be compressed by a lossy compression algorithm (e.g., JPEG). Normally a picture block contains more colours and less vertical or horizontal strokes than a text block. This combined characteristic is used to classify picture blocks.

\section{Text Block Prediction}

If the blocks located to the left and above the current block are both text blocks, the current block can be predicted as a text block. This is called text block prediction. This means that the predicted text block gets lossless compression applied even if it is not a text block. This is an effort to reduce classification time.

An experiment was designed to show the need of text block prediction, ten different computer screen images of size $1152 \times$ 1600 are used for the test, the result is shown in TABLE I.

In TABLE I, $N_{\text {text }}$ is the number of all text blocks, $N_{\text {prediction }}$ is the number of blocks which have top and left blocks as text blocks and so are candidates for prediction. Ratio is the result of dividing $N_{\text {prediction }}$ by $N_{\text {text }}$. Therefore $49.55 \%$ of text blocks in tested compound images could be predicted directly without classification using this algorithm.

If this text prediction method is applied to every block and a prediction error occurs, the error could be propagated to blocks around it. In order to avoid this situation and apply text prediction without error propagation, a pattern shown in Fig.6 is used to apply text prediction. In this pattern, blue

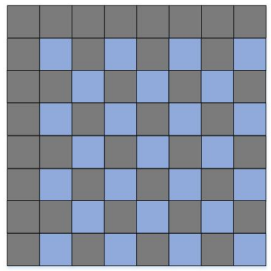

Figure 6. Proposed text block prediction pattern.

blocks can be predicted using text prediction and gray blocks will be classified using DWT or smooth prediction methods. In this pattern, every predicted text block is isolated from other predicted blocks to avoid error propagation. This gives a muchimproved accuracy of text block prediction even though the total percentage of predicted blocks is reduced. See results section.

\section{Smooth Block Prediction}

There is only one colour in a smooth block, so smooth blocks can be classified by counting the number of colours 
in the $16 \times 16$ block instead of using the DWT method. The processing time required to classify smooth blocks with colour counting is less than with the DWT method.

From experiments on compound images, there are many smooth blocks detected, e.g., blocks in website pages, background of WORD documents. In Fig.7, the blocks with green boundary are smooth blocks.

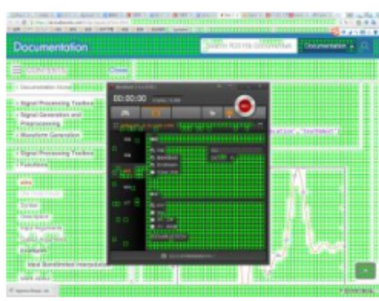

(a)

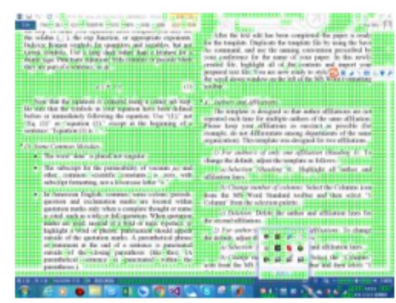

(b)
Figure 7. Smooth blocks in compound images (a) Website page (b) Word document

When the top and left blocks of the current block are smooth blocks, colour counting is applied to the current block to check if it is also a smooth block. If the number of colours is one, the current block will be classified as a smooth block, otherwise the DWT method is applied to it for further classification.

\section{E. Applying DWT method on $8 x 8$ down sampled block}

When a block of size $16 \times 16$ is down sampled to an $8 \times 8$ block, visual and texture characteristics of the original block are kept. The images in Fig.8 show the original blocks, of size $16 \times 16$, and the corresponding down sampled blocks, of size $8 \times 8$. The sampled blocks are rescaled.
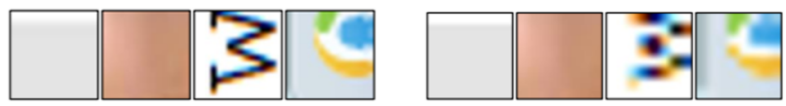

Figure 8. Blocks before (left) and after (right) down-sampling (magnified)

\section{F. Checking for picture blocks misclassified as text blocks}

If only the DWT method is applied for classification, some picture blocks can be misclassified as text blocks because they have a similar texture characteristic to text blocks. There are some examples shown in Fig.9.
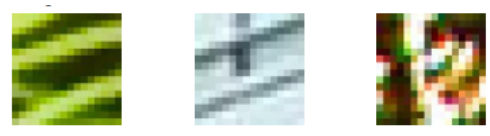

Figure 9. Blocks which are easily misclassified as text blocks when using the DWT method only

One key difference between text blocks and picture blocks is the number of colours in the block. Usually, a picture block contains many colours but a text block contains a small number of colours.

It is found that counting the number of colours in the horizontal or vertical direction within a block saves processing
Table II

NUMBER OF DIFFERENT TYPE BLOCKS USED FOR TEST

\begin{tabular}{|c|c|c|c|c|c|}
\hline$N_{\text {total }}$ & $N_{\text {smooth }}$ & $N_{\text {fuzzy }}$ & $N_{\text {sparse }}$ & $N_{\text {text }}$ & $N_{\text {pic }}$ \\
\hline 2000 & 400 & 400 & 400 & 400 & 400 \\
\hline
\end{tabular}

Table III

CLASSIFICATION ACCURACY OF DIFFERENT BLOCK TYPES

\begin{tabular}{|c|c|c|c|c|c|}
\hline Smooth & Fuzzy & Sparse & Text & Picture & Average \\
\hline $100 \%$ & $99.5 \%$ & $99.0 \%$ & $97.5 \%$ & $96.3 \%$ & $98.46 \%$ \\
\hline
\end{tabular}

time and is sufficiently accurate to double check the blocks that have been classified as text blocks by the DWT algorithm.

If a block is classified as a text block by the DWT method, and the number of colours, Nc, in specific horizontal or vertical lines (but not both) of this block is greater than a threshold value, Tc, then the block is re-classified as a picture block, otherwise it is classified as a text block.

\section{RESULTS}

The proposed algorithms are implemented on an Intel $\mathbb{R}$ Core $^{\mathrm{TM}}$ i5-4200M 2.50GHZ processor using Microsoft Visual Studio 2013 with $\mathrm{C}++$.

\section{A. Processing time and classification accuracy}

Text block and smooth block prediction along with downsampling are applied to reduce processing time. An experiment was designed to evaluate the accuracy of proposed classification method. Two-hundred smooth, sparse, fuzzy, picture and text blocks are selected manually as a test dataset. Table II shows the block numbers of different block types, $N_{\text {total }}$ is the number of all blocks. $N_{\text {smooth }}, N_{\text {fuzzy }}, N_{\text {sparse }}, N_{\text {text }}$ and $N_{\text {pic }}$ are the numbers of smooth, fuzzy, sparse, text and picture blocks. The classification accuracy of each block type is calculated using equation (2)

$$
\frac{\text { correctly detected blocks }}{\text { the total number of corresponding blocks }}
$$

The classification accuracy results are shown in Table III. The accuracy of classification on smooth blocks is $100 \%$ because every predicted smooth block will be checked by counting the colour number in this whole block.

42 compound images (height $=1152$, width $=1600$ ) are used to evaluate the classification time of the proposed method. These 42 images are taken from many application scenarios, such as web pages, opened Word documents, PowerPoint slides, computer desktop, etc. The experimental results of classification time are shown in Table IV and colour counting is applied to all test cases. In Table IV, OFF means this method or feature is not applied to the classification system. It is shown in Table IV that if only text prediction is applied to the 42 compound images (case 2), only $7.28 \%$ processing time is saved compared to case 1 . The reason is that only about $16 \%$ of all blocks are text blocks. Smooth block prediction saves $27.35 \%$ processing time (case 3 ) compared to case 2 . If both 
Table IV

PROCESSING TIME OF DIFFERENT CASES

\begin{tabular}{|c|c|c|c|}
\hline Case & Proposed method & Condition & Processing time (ms) \\
\hline \multirow{4}{*}{1} & Text prediction & OFF & \\
& Smooth prediction & OFF & 79.98 \\
& Down sampling & OFF & \\
\hline \multirow{3}{*}{2} & Text prediction & ON & \\
& Smooth prediction & OFF & 74.16 \\
& Down sampling & OFF & \\
\hline \multirow{3}{*}{3} & Text prediction & ON & \\
& Smooth prediction & ON & 53.88 \\
& Down sampling & OFF & \\
\hline \multirow{4}{*}{4} & Text prediction & ON & \\
& Smooth prediction & ON & 20.57 \\
& Down sampling & ON & \\
\hline
\end{tabular}

block prediction methods and down-sampling are applied (case 4), $74.28 \%$ of processing time is saved compared to case 1 . The processing time in Table IV represents the time it takes to classify an $1152 \times 1600$ compound image, it is an averaged value, $\mathbf{m s}$ is the time unit which represents millisecond.

The classification system presented in this paper is based on the DWT method proposed by Juliet. However, 8x8 is selected as the block size in his method and only two block types are used (i.e., text/graphics block and picture/background block). The average classification accuracy (i.e., 97.4\%) is stated in his paper but the classification time is not mentioned [11]

The colour counting idea presented in this paper is inspired by Tony Lin's paper[16]. He uses colour counting to perform coarse classification and the refinement is then applied to further classification. Blocks are classified into text blocks and picture blocks after coarse classification. The threshold value (i.e., $\mathrm{T}=32$ ) set for coarse classification is stated in his paper but the classification accuracy and time are not discussed.

The proposed classification system performance are compared to the methods proposed by Juliet and Tony Lin (coarse classification only). The classification methods proposed by Tony Lin and Juliet are re-implemented using $\mathrm{C}++$, the 200 text and 200 picture $16 \times 16$ blocks in Table II are used to test the accuracy of colour counting coarse classification proposed by Tony Lin. 42 compound images in previous experiment are used to test the classification time of methods proposed by Juliet and Tony Lin. The results are shown in Table V. The classification time in Table $\mathrm{V}$ represents the average time used to classify a compound image (height $=1152$, width $=1600$ ).

From Table V, it's observed that the proposed scheme has the highest classification accuracy compared with the other two methods. The classification time for each compound image of the proposed scheme is only $3.37 \mathrm{~ms}$ slower than the coarse classification method. The proposed scheme makes good tradeoffs between the other two classification methods because it not only retains high classification accuracy as DWT method proposed by Juliet but also reduces the time consumed on
Table V

CLASSIFICATION ACCURACY AND TIME OBTAINED USING THREE BLOCK CLASSIFICATION SCHEMES

\begin{tabular}{|c|c|c|c|}
\hline & $\begin{array}{c}\text { DWT by } \\
\text { Juliet }\end{array}$ & $\begin{array}{c}\text { Colour Counting } \\
\text { by Tony Lin }\end{array}$ & $\begin{array}{c}\text { proposed } \\
\text { Scheme }\end{array}$ \\
\hline Block Size & $8 \times 8$ & $16 \times 16$ & $16 \times 16$ \\
\hline $\begin{array}{c}\text { Average } \\
\text { Accuracy(\%) }\end{array}$ & 97.4 & 93.7 & 98.46 \\
\hline $\begin{array}{c}\text { Classification } \\
\text { Time(ms) }\end{array}$ & 90.5 & 17.2 & 20.57 \\
\hline
\end{tabular}

classification.

\section{B. Classification Result Example}

The classification result of a compound image with the proposed classification algorithm is shown in Fig.10. Blocks with green boundary are smooth blocks, blocks with gray boundary are sparse blocks, blocks with yellow boundary are fuzzy blocks, blocks with red boundary are picture blocks, and blocks with blue boundary are text blocks. This shows a near perfect classification of the complex compound image.

\section{CONClusion}

A very accurate and novel block classification method for compound images has been developed. In addition, novel methods have been used to reduce the classification processing time as shown in Fig. $3.16 \times 16$ is selected as the block size because it is optimum under a number of conditions. Blocks are classified into five types: smooth, sparse, fuzzy, picture and text. Block type prediction and down sampling are employed to reduce the classification time. The classification types of fuzzy and sparse blocks are unique to the proposed method.

In order to save classification processing time, spatial correlation of text blocks and smooth blocks is exploited to implement block prediction algorithms. The classification processing time saved by block prediction $32.63 \%$.

In order to save processing time on classification further, DWT is applied to down sampled blocks (i.e., 8x8 pixels) to find the texture characteristic rather than the original $16 \times 16$ pixel blocks. By combining these three methods, classification time is reduced by $74.28 \%$ compared to a non-optimised system.

The proposed classification scheme is compared to the schemes proposed by Juliet[18] and Tony Lin[16] and the results show that the proposed scheme has an improved overall performance taking into account classification accuracy and classification time.

With the novel classification methods and processing time reduction techniques, presented in this paper, the overall classification accuracy is $98 \%$ and the overall classification time is $20.57 \mathrm{~ms}$ average per frame. This represents a $74 \%$ improvement in processing time compared to [11].

\section{REFERENCES}

[1] Ricardo De Queiroz et al. "Mixed raster content (MRC) model for compound image compression". In: PROC. EI’99, VCIP, SPIE 3653 (1999), pp. 1106-1117. 


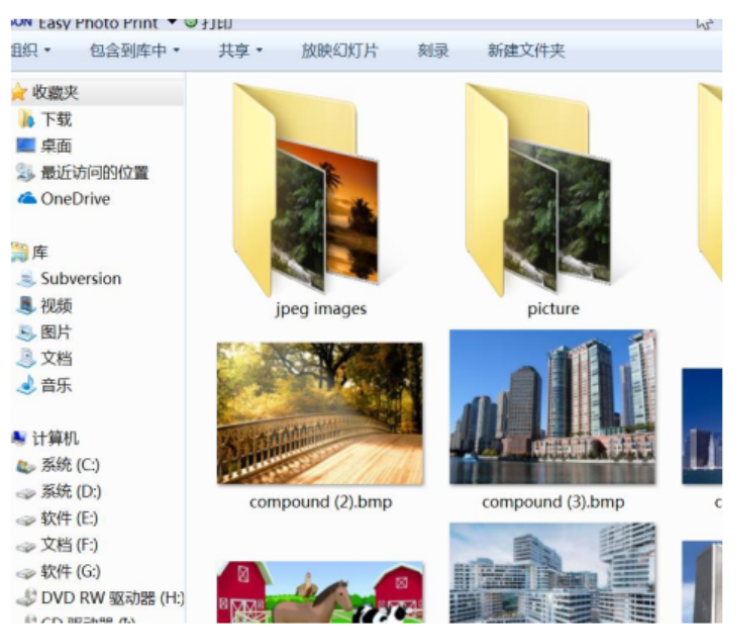

(a)

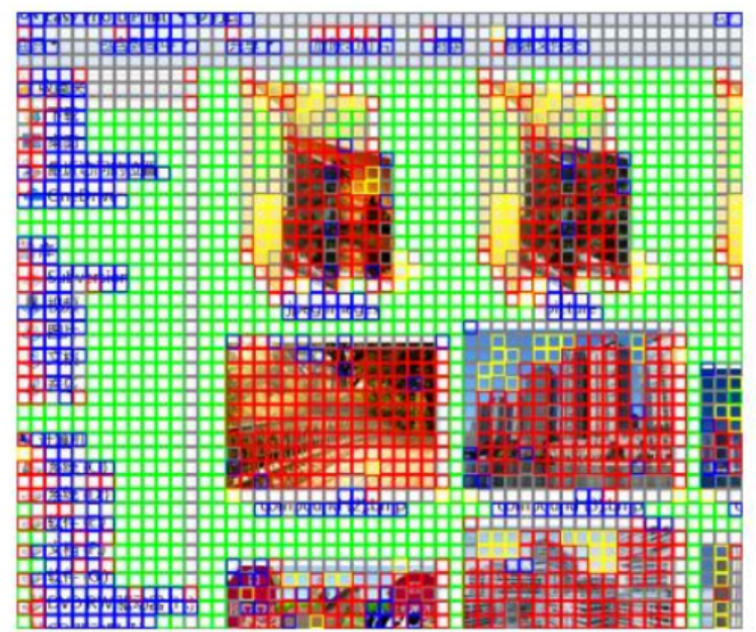

(b)

Figure 10. Classification result on computer screen. (a) Original image (b) Image after classification

[2] A. Said and A. Drukarev. "Simplified segmentation for compound image compression". In: Proceedings 1999 International Conference on Image Processing (Cat. 99CH36348). Vol. 1. IEEE, 1999, pp. 229-233. ISBN: 0-7803-5467-2. DOI: 10.1109/ICIP.1999.821603.

[3] Amir Said and Alex Drukarev. "Simplified Segmentation for Compound Image Compression". In: (1999), pp. 229-233.

[4] Xin Li and Shawmin Lei. "Block-based segmentation and adaptive coding for visually lossless compression of scanned documents". In: Proceedings 2001 International Conference on Image Processing 2 (2001), pp. 450-453. DOI: 10.1109/ICIP.2001.958148.

[5] Xin Li and Shawmin Lei. "Block-based segmentation and adaptive coding for visually lossless compression of scanned documents". In: Proceedings 2001 International Conference on Image Processing (2001), pp. 450-453. DOI: 10.1109/ICIP.2001.958148.

[6] T. Lin. "Compound image compression for real-time computer screen image transmission". In: IEEE Transactions on Image Processing 14.8 (Aug. 2005), pp. 9931005. ISSN: 1057-7149. DOI: 10.1109/TIP.2005.849776.

[7] Wenpeng Ding et al. "Block-based fast compression for compound images". In: 2006 IEEE International Conference on Multimedia and Expo, ICME 2006 Proceedings 2006 (2006), pp. 809-812. DOI: 10.1109/ ICME.2006.262624.

[8] S. E. Juliet, D. J. Florinabel, and V. Sadasivam. "Efficient coding of computer screen images with precise block classification using wavelet transform". In: Journal of the Institution of Engineers (India), Part CP: Computer Engineering Division 91.MAY (2010), pp. 17-23. ISSN: 09710469. DOI: 10.1049/iet-ipr.2009. 0237.

[9] Cuiling Lan, Guangming Shi, and Feng Wu. "Compress compound images in H.264/MPGE-4 AVC by exploit- ing spatial correlation". In: IEEE Transactions on Image Processing 19.4 (2010), pp. 946-957. ISSN: 10577149. DOI: 10.1109/TIP.2009.2038636.

[10] Iain E. Richardson and Dr Paul Anthony. H.264 Advanced Video Compression Standard (2). Wiley, 2010, p. 348. ISBN: 9780470516928.

[11] S Ebenezer Juliet and D Jemi Florinabel. "Efficient block prediction-based coding of computer screen images with precise block classification". In: 5.May 2010 (2011), pp. 306-314. DOI: 10.1049/iet-ipr.2009.0237.

[12] Ie Richardson. The H. 264 advanced video compression standard. Wiley, 2011, p. 346. ISBN: 9780470516928.

[13] Yanfei Shen et al. "Classification-Based Adaptive Compression Method for Computer Screen Image". In: 2012 IEEE International Conference on Multimedia and Expo Workshops. IEEE, July 2012, pp. 7-12. ISBN: 9781-4673-2027-6. DOI: 10.1109/ICMEW.2012.9.

[14] Yanfei Shen et al. "Classification-based adaptive compression method for computer screen image". In: Proceedings of the 2012 IEEE International Conference on Multimedia and Expo Workshops, ICMEW 2012 (2012), pp. 7-12. DOI: 10.1109/ICMEW.2012.9.

[15] Zhaotai Pan et al. "A low-complexity screen compression scheme for interactive screen sharing". In: IEEE Transactions on Circuits and Systems for Video Technology 23.6 (2013), pp. 949-960. ISSN: 10518215. DOI: 10.1109/TCSVT.2013.2243056.

[16] Pham Xuan Qui et al. "A Novel Efficient Approach for Screen Image Classification in Remote Display Protocol”. In: 2015 15th IEEE/ACM International Symposium on Cluster, Cloud and Grid Computing. IEEE, May 2015, pp. 1217-1220. ISBN: 978-1-4799-8006-2. DOI: 10.1109/CCGrid.2015.54. 